\title{
FOCUS: Frailty Management Optimisation through EIPAHA Commitments and Utilisation of Stakeholders' Input - an innovative European Project in elderly care*
}

ANTONIO CANO ${ }^{1, E, F}$, DONATA KURPAS ${ }^{2, E, F}$, MARIA MAGDALENA BUJNOWSKA-FEDAK ${ }^{2, E, F}$, SILVINA SANTANA ${ }^{3, E, F}$, CAROL HOLLAND ${ }^{4, E, F}, M_{A U R A} M_{A R C U C C I}^{5, E, F}$, ANA GONZALEZ-SEGURA ${ }^{6, \mathrm{E}, \mathrm{F}}$, MIRIAM VOLLENBROEK-HUTTEN ${ }^{7,8, \mathrm{E}, \mathrm{F}}$, BARBARA D'AVANZO ${ }^{9, \mathrm{E}, \mathrm{F}}$, ALESSANDRO

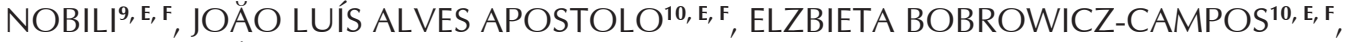
ANA M. MARTÍNEZ-ARROYO ${ }^{11, E, F}$

\author{
${ }^{1}$ Universitat de Valencia, Spain \\ ${ }^{2}$ Family Medicine Department, Wroclaw Medical University, Poland \\ ${ }^{3}$ University of Aveiro, Portugal \\ ${ }^{4}$ Aston Research Centre for Healthy Ageing (ARCHA), Aston University, UK \\ ${ }^{5}$ Fondazione Cá Granda Ospedale Maggiore Policlinico \& University of Milan, Milan, Italy \\ ${ }^{6}$ Everis Spain S.L.U., Spain \\ ${ }^{7}$ Roessingh Research and Development (RRD), the Netherlands \\ ${ }^{8}$ University of Twente, Faculty of Electrical Engineering, Mathematics and Computer Science, \\ Telemedicine group, the Netherlands \\ ${ }^{9}$ IRCCS Istituto di Ricerche Farmacologiche Mario Negri, Milan, Italy \\ ${ }^{10}$ Nursing School of Coimbra, Portugal \\ ${ }^{11}$ ESAM Tecnología, S.L., Spain
}

A - Study Design, B - Data Collection, C - Statistical Analysis, D - Data Interpretation, E - Manuscript Preparation, $\mathbf{F}$ - Literature Search, $\mathbf{G}$ - Funds Collection

Summary The goal of FOCUS, which stands for Frailty Management Optimization through EIPAHA Commitments and Utilization of Stakeholders' Input, is to reduce the burden of frailty in Europe. The partners are working on advancing knowledge of frailty detection, assessment, and management, including biological, clinical, cognitive and psychosocial markers, in order to change the paradigm of frailty care from acute intervention to prevention. FOCUS partners are working on ways to integrate the best available evidence from frailty-related screening tools, epidemiological and interventional studies into the care of frail people and their quality of life. Frail citizens in Italy, Poland and the UK and their caregivers are being called to express their views and their experiences with treatments and interventions aimed at improving quality of life. The FOCUS Consortium is developing pathways to leverage the knowledge available and to put it in the service of frail citizens. In order to reach out to the broadest audience possible, the FOCUS Platform for Knowledge Exchange and the platform for Scaling Up are being developed with the collaboration of stakeholders. The FOCUS project is a development of the work being done by the European Innovation Partnership on Active and Healthy Ageing (EIPAHA), which aims to increase the average healthy lifespan in Europe by 2020 while fostering sustainability of health/social care systems and innovation in Europe. The knowledge and tools developed by the FOCUS project, with input from stakeholders, will be deployed to all EIPAHA participants dealing with frail older citizens to support activities and optimize performance.

Key words: frailty, stakeholders, healthy ageing.

Fam Med Prim Care Rev 2016; 18(3): 373-376

\section{Background}

The FOCUS (Frailty Management Optimisation through EIPAHA Commitments and Utilisation of Stakeholders' Input) project aims to critically reduce and prevent the burden of frailty in Europe by supporting development of the commitments that focus on early diagnosis/screening and on management of frailty within the European Innovation Partnership for Active Healthy Ageing (EIPAHA). Prevention, early diagnosis and management of frailty pose a series of challenges, which add to the many gaps still existing in the fluid exchange with stakeholders.

The EIPAHA was selected because it constitutes a critical instrument for the $\mathrm{EU}$ and because it composes the widest representation of initiatives regarding frailty in Europe. Facilitation of exchange of best practice and the support of networks for knowledge sharing have been taken as objectives in the project.

\footnotetext{
* This article is part of the project/joint action '664367/FOCUS' which has received funding from the European Union's Health Programme (2014-2020) and Ministry of Science and Higher Education in Poland (funding in years 2015-2018 allocated for the international co-financed project).
} 


\section{Frailty and ageing}

Ageing is understood as a biological process resulting from the gradual, lifelong accumulation of molecular and cellular damage [1]. This burden produces a clinical phenotype where both frailty and disease are common outcomes.

Frailty is defined as a state of high vulnerability for adverse health outcomes when exposed to a stressor [2]. As a consequence, frailty associates with disease, dependency and death that, among other adverse and obvious outcomes, entail a high demand and utilization of health and community services and other resources [3-8]. Although there is no unique conceptualization of frailty [9-11], the two most widely accepted models identify an overlapping population at high risk of poor prognosis [12-13]. Depending on the definition selected, estimates of frailty vary from $4 \%$ to $17 \%$ [14].

The impact of ageing is malleable, as is frailty, which is seen as a continuum with a pre-frail state identified [2]. Evidence suggests that lifestyle and other interventions may help to slow down the ageing impacts. There is therefore scientific consensus that, given both high prevalence and impact on health, screening and prevention for frailty at population level is recommended [15].

Some risk factors have been associated with frailty. Polypharmacy, for example, has been recognized as an independent risk factor for development of a vulnerable state [1618]. Indeed, focus on frailty has been the driver for initiatives aiming at the modification of healthcare systems. A change from an organization focused around single-system illnesses (disease oriented approach) towards a more holistic viewpoint of the patient (patient oriented approach), involving coordinated health and social interventions, would result.

However, there are many uncertainties around the strategies to identify and tackle frailty. Together with the different approaches in screening and diagnosis, there is insufficient information on the regulatory pathophysiological pathways, and there is a lack of models to assess the value of the impact on health economy.

Finally, there is a deficiency in personalised integrated solutions. Together, these uncertainties at present translate into a lack of effective management guidelines.

The need for a comprehensive consideration of the main clinical conditions affecting ageing is reflected by the 2014 Annual Work Programme for the implementation of the third Programme of the Union's action in the field of health (2014-2020), where frailty, multi-chronic conditions (multimorbidity), and integrated care are placed within the same action under thematic priority [19].

There is a growing interest in frailty. The search engine PubMed for this key-word shows an exponential increase of titles, accumulating in the last 10 years. The body of knowledge regarding this conception is represented in the form of systematic reviews or other similar forms of scientific literature, which provide the evidence-based practice available at the present time.

As detailed later, a principal objective of the FOCUS project is to consolidate the up-to-date evidence-based information about frailty, together with the related measures and methods.

\section{Frailty in the European Union}

The Innovation Union strategy [19] was launched by the European Commission to face major societal challenges claiming for innovation emergency. After specific scrutiny, some dysfunctions have been detected by an ad hoc created Independent Expert Group, which has recommended a list of interventions [20]. Particularly: an ecosystem approach facilitating the implementation of innovation and the collaboration between actors as well as the integration of new actors, more effective sharing of knowledge and the identifica- tion of appropriate indicators capable of effectively tracking the overall development, or improvement of the stakeholder engagement to ease synergies or policy maker commitment.

European Innovations Partnership (EIP) was created to establish a forum for exchange and to improve cooperation between actors, but dysfunctions have been detected. Commissioned by the Union, the Expert Group [2] recommended a new ecosystem for innovation and scaling-up, where optimisation will include improvements in knowledge exchange, and where a collaborating platform will facilitate synergies. Also, strengthening the bottom-up approach, with a definitive reinforcement in the participation of partners and stakeholders, should combine with the necessary topdown leadership. Finally, the need for continual monitoring and evaluation will require the development of appropriate indicators and methodology.

EIPAHA was launched in May 2011. Since then, almost 600 commitments have been submitted by an array of stakeholders. Frailty has been specifically tackled in the action groups. For example, one of the action groups, A3, specifically focuses in the prevention of functional decline and frailty.

Together with monitoring, much of the activity in the Action Groups has concentrated in promoting exchange for speeding up progression. The initiatives to advance in that regard have substantiated into: the introduction of common tasks, the use of a social network for facilitating contact, groups meetings with responsible Commission officers, annual conferences and specific inter-groups activities.

The key factors described by the experts and promoted within EIPAHA, all mentioned above, define the basis for optimisation, in which this project is based.

\section{Objectives of the Project}

The main goal of FOCUS project is to contribute to the reduction of the burden created by frailty by introducing innovative practices leading to optimization of the performance of EIPAHA.

The following specific objectives have been defined:

1. To consolidate the available evidence regarding screening and interventions for frailty and pre-frailty from the published literature.

2. To gain knowledge and understanding of the needs of stakeholders (including citizens, patients, carers, providers, policy makers, etc.), regarding frailty: care, social support, prevention.

3. To examine critically the reality of the commitments in EIPAHA that deal with frailty screening, prevention or management.

4. To identify possible targets for customized strategies aimed at optimization and scalability within EIPAHA through an analytical and standardized characterization and comparison of EIP AHA interventions. This will focus on common indicators and the projections of their impact on resource consumption at population-level.

5. To develop guidelines as a customized service to aid implementation, optimization and scalability supported by the functionality of an ICT platform.

6. To create a network representative of European diversity, including a balanced representation of partners in commitments and stakeholders representative of European diversity.

7. To deploy the PKE (Platform for Knowledge Exchange), a technological platform to support the remote access and scaling up of the good practice modules resulting from this project.

8. To assess performance of the generated guidelines through a test performed on the set of EIPAHA commitments that are being run by partners in the consortium. 


\section{Methodology}

Several approaches are combined in the framework of a participatory research design. Quantitative approaches will be used in two systematic reviews, one related to reliability, validity and ability to predict adverse health outcomes of existing frailty tools, another about the effectiveness of interventions in preventing the progression pre-frailty and frailty. Moreover quantitative approaches will be used in the analyses of the characteristics of the activities represented in the EIP-AHA, comparative effectiveness analyses, and in the use of models predictive of resource consumptions according to needs. A qualitative approach will be used for the metasynthesis of several stakeholders' opinions and experiences about screening and management of frailty and in the five focus groups with different stakeholders conducted in three countries. Also, a mixed-method approach will be used to develop indicators of quality of the EIP-AHA activities will be developed with a mixed-method approach since they will be derived from several sources, including the focus groups findings, and a Delphi for the final selection.

Finally, stakeholders will be involved in the definition of the characteristics of the platform for knowledge exchange through surveys collecting their needs and preferences, and suggestions. To specify, the tools employed are:

- Quality appraisal resulting in final sets of papers for analyses and draft of conclusions and recommendations (objective 1, objective 2);

- Focus groups meetings including stakeholders: frail and non-frail older adults, caregivers, health and social care professionals, (objective 2);

- $\quad$ Structured surveys (objective 3);

- Delphi consensus (objective 4);

- Skype conferences (objective 4, objective 5, objective 6 , objective 7 , objective 8 );

- Email contacts (objective 4, objective 7);

- On site meetings (objective 6, objective 7, objective 8);

- Virtual meetings of the network (objective 8).

\section{Target groups}

The FOCUS Consortium has identified four levels of targets groups of its activities. The first, immediate target group is constituted of the growing population of ageing people within the EU, together with their caregivers. The second, the stakeholders, who will be given a channel to directly express their needs at a European scale. In addition to patients and their caregivers, already considered preferentially as the first target, health care professionals, knowledge institutes, SMEs, service providers, insurance companies, policy makers, are further examples of this group. The third are the partners dealing with frailty screening and management within the specific sector in EIPAHA. A substantial improvement in the respective performance of the affected commitments will ensue.

The fourth target group is constituted by the Member States. Advances having an impact on citizens affected by frailty will result in real consolidation of profiles where empowerment of citizens, sustainability and trans-European standardization will become a more prevalent reality. The corresponding impact on cross-border issues will ensure this becomes a more permanent reality across Europe.

\section{Political relevance}

In consistence with the EU Regulation No 282/2014 of the European Parliament and of the Council of March 2014, and of the Commission White Paper "Together for Health: A strategic approach for the EU 2008-2013", the Work Program stresses four specific objectives, the first being the promotion of health, and the third being the contribution to innovative, efficient and sustainable health systems.

The FOCUS project contributes to health promotion, as clearly stated throughout the previous pages; it is innovative, because it addresses gaps in early diagnosis and associated frailty management in Europe; it promotes the optimisation of the work being conducted by existing commitments, by deploying appropriate instruments and fostering a prioritised participation of stakeholders, leading to the spread of customised guidelines to improve performance developed by the FOCUS project with stakeholders input; it is compatible with existing actions: for example, scalability will be based on the knowledge being generated by a FP7 funded project, Perssilaa (www.perssilaa.eu); it participates in networks funded under the EU Health Programs, since seven of the partners in the consortium are members of EIPAHA.

According to Eurostat Population Projections, the number of people aged $65+$ in the EU will grow by $70 \%$ by 2050 [19]. Frailty dramatically ruins what otherwise would be healthy ageing, and this is a first level public health issue. Consequently, the added value in public health of the FOCUS project derives from: the impact on the target group, frail and pre-frail citizens in this case and the multiplier effect, expected through the improvement in the performance of EIPAHA commitments. EIPAHA constitutes a huge network whose impact is extraordinary. Accordingly, the project will act as a chain reaction, due to the amplifying power constituted by EIPAHA.

The closeness to the needs expressed by stakeholders will be expressed in the generation of the database for analysis and will be permanently represented in the network. Among them, policy makers represent an enormous impact potential (macro-level). Vice versa, the inclusion of final users (caregivers, non-government organisations, patients associations, etc.) warrants the bottom-up approach. The favorable reception of these organisations in public opinion plus the enormous impact of volunteerism offer a powerful catalytic effect.

Finally, the FOCUS project helps partners in EIPAHA, and in that sense, promotes best practice, creates benchmarking for decision making, strengthens networking, manages in a particularly excellent way the cross border issues, and because it finally helps the EIPAHA to implement EU legislation.

\section{Pertinence of geographical coverage}

The actual consortium constitutes an equilibrated and large representation of the geography in the European Union. Centre-North (the Netherlands) and South (Spain and Italy), East (Poland) and West (UK, Portugal) have a representation. This large representation makes it possible to include different profiles of citizens with regards to pre-frailty and frailty, which has implications in screening and management. Frailty/prefrailty is a pressing need all over Europe, but has different faces in the South as compared with the North, for example.

The European Union is a contrasting reality, with differences affecting life expectancy (LE) and healthy-life years $(\mathrm{HLY})$. The FOCUS project is sensitive to the cultural diversity and the cross border issues because it works with partners and commitments from all over Europe. The views, social contexts, customs, local policies, and other related matters are adequately represented. Moreover, the use of real time Information and Communication Technologies (ICTs) may speed up the optimisation in practices and the corresponding benefit for citizens, even in Member States undergoing economic crises and reduction in available resources. 


\section{Expected outcomes}

The deliverables of the FOCUS project will offer significant potential improvements for innovative, coordinated and comprehensive community based prevention with regard to the optimization of functional capacity tools to address pre-frailty, and for improving the management of frailty. In doing that the project focuses on EIPAHA as the European level forum where is a solid representation of partners and stakeholders.

Specifically:

- It will provide a detailed appraisal of those most relevant variables to take into account in the process of building the most suitable strategy, i.e. the state of the art, the reality of commitments working in the field within EIPAHA, and the needs expressed by stakeholders.

- It will evaluate the appropriateness of strategies identified through newly generated guidelines. Relevant, responsive, and scientifically sound indicators will help in the process.

- It will create a technological platform for perpetuating the fruitful exchange between EIPAHA partners and stakeholders, the Platform for Knowledge Exchange. Other functionalities repository, etc.) will amplify the utility of the platform.

Finally, it will test guidelines for impact in the real world represented by commitments run by partners in the consortium. Feedback from the experience will help to further optimise guidelines.

Source of funding: This paper is part of the project /joint action '664367/ FOCUS' which has received funding from the European Union's Health Programme (2014-2020) and Ministry of Science and Higher Education in Poland (funding in years 2015-2018 allocated for the international co-financed project).

Conflict of interest: The authors declare no conflict of interests.

\section{References}

1. Kirkwood TB. Understanding the odd science of aging. Cell 2005; 120(4): 437-447.

2. Clegg A, Young J, Iliffe S, et al. Frailty in elderly people. Lancet 2013; 381(9868): 752-762.

3. Fried LP, Tangen CM, Walston J, et al. Frailty in older adults: evidence for a phenotype. J Gerontol A Biol Sci Med Sci 2001; 56(3): M146-M156.

4. Rockwood K, Mitnitski A, Song X, et al. Long-term risks of death and institutionalization of elderly people in relation to deficit accumulation at age 70. J Am Geriatr Soc 2006; 54(6): 975-979.

5. Auyeung TW, Lee JS, Kwok T, et al. Physical frailty predicts future cognitive decline: a four-year prospective study in 2737 cognitively normal older adults. J Nutr Health Aging 2011; 15(8): 690-694.

6. Hurria A, Togawa K, Mohile SG, et al. Predicting chemotherapy toxicity in older adults with cancer: a prospective multicenter study. J Clin Oncol 2011; 29(25): 3457-3465.

7. Makary MA, Segev DL, Pronovost PJ, et al. Frailty as a predictor of surgical outcomes in older patients. J Am Coll Surg 2010; 210(6): 901-908.

8. Hastings SN, Purser JL, Johnson KS, et al. Frailty predicts some but not all adverse outcomes in older adults discharged from the emergency department. J Am Geriatr Soc 2008; 56(9): 1651-1657.

9. Fried LP, Ferrucci L, Darer J, et al. Untangling the concepts of disability, frailty, and comorbidity: implications for improved targeting and care. J Gerontol A Biol Sci Med Sci 2004; 59(3): 255-263.

10. Sternberg SA, Wershof Schwartz A, Karunananthan S, et al. The identification of frailty: a systematic literaturę review. J Am Geriatr Soc 2011; 59(11): 2129-2138.

11. Rockwood K, Mitnitski A. Frailty defined by deficit accumulation and geriatric medicine defined by frailty. Clin Geriatr Med $2011 ; 27(1): 17-26$.

12. Kulminski A, Ukrainsteva SV, Kulminskaya IV, et al. Cumulative deficits better characterize susceptibility to death in elderly people than phenotypic frailty: lessons from the Cardiovascular Health Study. J Am Geriatr Soc 2008; 56(5): 898-903.

13. Theou O, Brothers TD, Mitntiski A, et al. Operationalization of frailty using eight commonly used scales and comparison of their ability to predict all-cause mortality. J Am Geriatr Soc 2013; 61(9): 1537-1551.

14. Collard RM, Boter H, Schoevers RA, Oude Voshaar RC. Prevalence of frailty in community-dwelling older persons: a systematic review. J Am Geriatr Soc 2012; 60:1487-1492.

15. Morley JE, Vellas B, van Kan GA et al. Frailty consensus: a call to action. J Am Med Dir Assoc 2013;14(6): 392-397.

16. Gnjidic D, Hilmer SN, Blyth FM, et al. Polypharmacy cutoff and outcomes: Five or more medicines were used to identify community-dwelling older men at risk of different adverse outcomes. J Clin Epidemiol 2012; 65(9): 989-995.

17. Flaherty JH, Perry HM 3rd, Lynchard GS, et al. Polypharmacy and hospitalization among older home care patients. J Gerontol A Biol Sci Med Sci 2000; 55(10): M554-M559.

18. Nobili A, Licata G, Salerno F, et al. SIMI Investigators. Polypharmacy, length of hospital stay, and in-hospital mortality among elderly patients in internal medicine wards. The REPOSI study. Eur J Clin Pharmacol 2011; 67(5): 507-519.

19. Innovation Union. A Europe 2020 Initiative [cited 15.09.2014]. Available from URL: http://ec.europa.eu/research/innovation-union/index en.cfm?pg=home.

20. Outriders for European Competitiveness. European Innovation Partnerships (EIPs) as a Tool for Systemic Change. Report of the Independent Expert Group [cited 15.09.2014]. Available from URL: http://ec.europa.eu/research/innovation-union/pdf/outriders_for_european_competitiveness_eip.pdf\#view=fit\&pagemode=none.

Address for correspondence:

Donata Kurpas, MD, PhD, Assoc Prof.

Katedra i Zakład Medycyny Rodzinnej UM

ul. Syrokomli 1

51-141 Wrocław

Polska

Tel.: +48 606 323-449

Received: 23.04.2016

E-mail:dkurpas@hotmail.com 